\title{
Treatment Patterns and Outcomes for Patients with Adrenocortical Carcinoma Associated with Hospital Case Volume in the United States: A Reply
}

\author{
Lauren Gratian, MD
}

Duke University, Durham, NC

\section{TO THE EDITORS:}

We appreciate the invitation to respond to the letter by Mallin et al. (http://www.surgonc.org/news-publications/ annals-of-surgical-oncology/letters-to-the-editor/treatmentpatterns-and-outcomes-for-patients-with-adrenocorticalcarcinoma-associated-with-hospital-case-volume-in-theunited-states), and thank the authors for their clarification regarding the missing percentages reported in our study from the National Cancer Data Base (NCDB). ${ }^{1}$ We understand that the NCDB continues to add new variables as they become available. Results for American Joint Commission on Cancer staging, Charlson-Deyo comorbidity scores, presence of distant metastases, and the surgical approach variables were only reported for a portion of the cases of adrenocortical cancer recorded in the NCDB. In an effort to maintain statistical power, we elected to include all cases from 1998 through 2011, rather than limiting our analysis to only those cases for which all variables were available. Table 1 provided a descriptive account of the variables analyzed for all cases in the study. We recognize that the percentage of missing data for variables would be much lower if we had included only the years in which they were reported. Despite some limitations of the NCDB, our study provides unique and valuable information at a national level for adrenocortical carcinoma patients.

\section{REFERENCES}

1. Gratian L, Pura J, Dinan M, et al. Treatment patterns and outcomes for patients with adrenocortical carcinoma associated with hospital case volume in the United States. Ann Surg Oncol. 2014;21(11):3509-14. 